\section{Simultaneidade de fatores de risco para doenças crônicas não transmissíveis entre idosos da zona urbana de Pelotas, Rio Grande do Sul, Brasil}

\author{
Simultaneity of risk factors for chronic \\ non-communicable diseases in the elderly \\ in Pelotas, Rio Grande do Sul State, Brazil
}

Simultaneidad de factores de riesgo para enfermedades crónicas no transmisibles entre ancianos de la zona urbana de Pelotas, Río Grande do Sul, Brasil

\section{Resumo}

O objetivo do estudo foi descrever a simultaneidade de fatores de risco para doenças crônicas não transmissíveis em idosos (60 anos ou mais), residentes em uma cidade do Sul do Brasil. Trata-se de um estudo transversal, de base populacional, realizado em 1.451 idosos em 2013. Com uma abordagem de análise de clusters, foi avaliado o agrupamento entre os fatores de risco em estudo (tabagismo, consumo de álcool, excesso de peso e inatividade física). Para a avaliação da associação da simultaneidade dos fatores de risco com variáveis sociodemográficas, foi utilizada regressão logística. O agrupamento mais frequente entre os homens $(18,1 \%)$ e mulheres $(30,7 \%)$ foi inatividade física + excesso de peso. As combinações consumo de álcool + excesso de peso excederam o esperado entre os homens $(O / E=1,27$; IC95\%: 1,$01 ; 1,59)$ e mulheres $(O / E=1,72 ;$ IC95\%: 1,$35 ; 2,20)$. A presença de dois ou mais fatores de risco na população idosa $(88,1 \%)$ aponta para a necessidade de intervenções específicas para esta população voltadas ao combate simultâneo dos fatores de risco e não de forma isolada.

Doença Crônica; Fatores de Risco; Idoso

\author{
Maurício Feijó da Cruz 1 \\ Virgílio Viana Ramires 1 \\ Andrea Wendt 1 \\ Grégore Iven Mielke 1 \\ Jeovany Martinez-Mesa 2 \\ Fernando César Wehrmeister 1
}

doi: 10.1590/0102-311X00021916

\author{
Correspondência \\ F. C. Wehrmeister \\ Programa de Pós-graduação em Epidemiologia, Universidade \\ Federal de Pelotas. \\ Rua Marechal Deodoro 1160, 3o andar, Pelotas, RS \\ 96020-220, Brasil. \\ fcwehrmeister@yahoo.com.br \\ 1 Universidade Federal de Pelotas, Pelotas, Brasil. \\ 2 Faculdade Meridional, Passo Fundo, Brasil.
}




\section{Introdução}

Os processos de transição demográfica e epidemiológica, no Brasil, ocorreram de forma acelerada quando comparados aos países de renda alta 1,2. Essas duas transições contribuíram para quadruplicar a população brasileira idosa nos últimos 60 anos ${ }^{3}$. As projeções do Instituto Brasileiro de Geografia e Estatística (IBGE) estimam que em 2030, o percentual de indivíduos com 65 anos ou mais no Brasil, chegue a $13,4 \%(\mathrm{~N}=29.899 .007)$ da população geral. Da mesma forma, a expectativa de vida que em 2016 é de 75,7 anos, em 2030 poderá chegar a 78,6 4. Isso acarreta uma consequente mudança na carga de morbimortalidade do país, passando de um perfil com predominância de doenças infecciosas e parasitárias para um cenário com predomínio de doenças crônicas não transmissíveis (DCNT) 2 . Tal cenário de crescimento das DCNT é ainda mais preocupante na população idosa, especialmente em função das alterações biológicas e sociais intrínsecas ao envelhecimento 5,6.

Entre os fatores de risco mais importantes para o aumento da carga de DCNT estão as mudanças nos padrões de comportamento da população, dentre os quais se destacam a inatividade física 7, o excesso de peso, o consumo abusivo de álcool 8,9 e o tabagismo 10,11. Mundialmente, a exposição ao tabaco é responsável por quase seis milhões de mortes, enquanto a inatividade física é responsável por cerca de cinco milhões por ano 12 . São atribuídos ao excesso de peso 2,8 milhões de óbitos anuais, semelhante ao que é atribuído ao consumo de álcool (2,3 milhões) 13. No Brasil, estimou-se que, em 2003, 13,6\% das mortes de adultos de 16 capitais brasileiras foram atribuídas ao tabagismo. Já para o consumo de álcool, a taxa de mortalidade foi de 5,17 por 100 mil pessoas em 2007 14. Atualmente, ainda no Brasil, de acordo a Pesquisa Nacional de Saúde, 46\% da população é insuficientemente ativo (não atinge os 150 minutos semanais), 14,5\% fumantes, 13,7\% fazem consumo abusivo de álcool e apenas $37,3 \%$ consomem frutas e hortaliças 15 . Estudo realizado em capitais do país apontou que mais da metade dos brasileiros (50,8\%) tem excesso de peso 16 .

Estudos sobre prevalência de fatores de risco isoladamente são relativamente abundantes na literatura científica. Todavia, estudos sobre a prevalência simultânea desses fatores ainda são menos frequentes, mesmo sabendo que as múltiplas causas são importantes para o desenvolvimento de DCNT 17,18. Além disso, a maioria dos estudos que avaliaram simultaneidade de fatores de risco foi realizada em populações de adultos e adolescentes 19,20,21,22,23,24 e menos frequentemente em população idosa. Um estudo realizado com idosos de Goiânia, Goiás, atendidos pelo Sistema Único de Saúde (SUS) identificou que mais de $80 \%$ da amostra apresentava mais que um fator de risco 25 .

Sendo assim, há a necessidade de estudos que identifiquem e expliquem as combinações mais frequentes dos fatores de risco em idosos, uma vez que a ocorrência simultânea e os agrupamentos podem variar de acordo com condições sociodemográficas tais como idade, cor de pele, nível socioeconômico entre outras 19,21,23. Nesse sentido, este estudo tem por objetivos descrever a ocorrência simultânea desses fatores de risco na população com 60 anos ou mais, identificando como ocorrem os agrupamentos entre eles, além de testar associações entre o acúmulo de tais fatores de risco e características sociodemográficas da população.

\section{Métodos}

Trata-se de um estudo transversal de base populacional, inserido em um consórcio de pesquisa 26 realizado pelo Programa de Pós-graduação em Epidemiologia, Universidade Federal de Pelotas (UFPel), realizado na zona urbana do Município de Pelotas, localizado ao sul do Estado do Rio Grande do Sul. O município possui 328.275 habitantes, dos quais 46.099 são idosos 27 . A população-alvo foi composta por indivíduos com 60 anos ou mais, não institucionalizados e residentes na zona urbana de Pelotas.

Para o cálculo de tamanho da amostra, foi considerada uma prevalência de dois ou mais fatores de risco de $35 \%$ com estimativa de erro de quatro pontos percentuais e intervalo de $95 \%$ de confiança (IC95\%), resultando em 984 indivíduos, acrescidos de 20\% para possíveis perdas e recusas. Em razão do formato de consórcio de pesquisa, para a coleta de dados, a estimativa de tamanho de amostra adotada foi de 1.649 idosos, o maior número entre os desfechos estudados.

O processo de seleção amostral foi realizado em dois estágios: setor censitário e domicílio. De acordo com o censo de 2010, o município pesquisado possui 488 setores censitários 27, dos quais 
19 setores foram reagrupados, por possuírem menos de 15 idosos, totalizando 469 setores. Inicialmente, foram selecionados 133 setores censitários e, posteriormente, para obter o número de domicílios necessários para encontrar o tamanho amostral necessário foram selecionados 31 domicílios em cada um dos 133 setores amostrados. Dessa forma, foi possível a identificação de pelo menos 12 idosos por setor. Todos os domicílios em cada um dos setores selecionados foram listados e sorteados sistematicamente.

\section{Fatores de risco}

Para esse estudo foram definidos quatro fatores de risco: tabagismo, consumo de álcool, excesso de peso e inatividade física, que foram coletados e operacionalizados conforme descrição a seguir. O fumo foi coletado por meio da questão “ $O(A)$ senhor(a) fuma ou já fumou?”. Se o entrevistado afirmar que fuma/fumava menos de um cigarro por dia por período inferior a 30 dias, não foi considerado fumante/ex-fumante 28. O consumo de álcool foi avaliado pela pergunta "Nos últimos 30 dias o senhor consumiu alguma bebida que tenha álcool?"; aqueles que responderam "sim" a essa pergunta foram considerados como consumidores de álcool.

Para avaliação do excesso de peso, foi utilizado o critério estabelecido por Lipschitz 29, em que indivíduos com índice de massa corporal (IMC) $>27 \mathrm{~kg} / \mathrm{m}^{2}$ foram considerados com excesso de peso (sobrepeso, obesidade). O IMC foi obtido pela divisão do peso em quilogramas pelo quadrado da altura em metros $\left(\mathrm{kg} / \mathrm{m}^{2}\right)$. A medida de peso foi obtida com utilização de balanças eletrônicas da marca Tanita, modelo UM-080 (Tanita Corporation, Arlington Heights, Estados Unidos), com capacidade máxima de $150 \mathrm{~kg}$ e precisão de $100 \mathrm{~g}$. Foi utilizada a medida de altura de joelho com o idoso sentado, pelo uso de antropômetro infantil em madeira da marca Indaiá (Indaiá Bengalas, Indaiatuba, Brasil), com escala de $100 \mathrm{~cm}$, graduação em milímetros e numerada a cada centímetro. Para estimar a altura a partir da altura do joelho, foram utilizadas as equações propostas por Chumlea \& Guo 30. Esse método apresenta acurácia suficiente para mensuração de altura ortostática em idosos 31, já que ela é difícil de ser mantida em idosos.

A inatividade física foi avaliada pelo International Physical Activity Questionnaire (IPAQ) 32, nas seções de lazer e deslocamento. Todos os indivíduos que não atingiram 150 minutos semanais de atividades físicas, de acordo com a recomendação da Organização Mundial da Saúde (OMS) 33, foram considerados inativos.

\section{Análises estatísticas}

Para a prevalência de múltiplos fatores foi criado um escore de 0 a 4, com a atribuição de um ponto para cada fator apresentado, sendo 0 a ausência e 4 a presença de todos os fatores de risco.

Ao avaliar a simultaneidade, uma abordagem de análise de clusters foi utilizada, na qual se considera a razão entre as prevalências observadas e esperadas $(\mathrm{O} / \mathrm{E})$ para cada agrupamento. A prevalência esperada para cada combinação específica é calculada multiplicando a prevalência dos fatores de risco presentes pelo inverso da prevalência dos fatores ausentes. Por exemplo, para calcular um cluster de inatividade física (I), tabagismo (T) e consumo de álcool (A), a multiplicação se dará da seguinte forma: I x T x A x (1-E), onde: I é a prevalência de inatividade física, T é a prevalência de tabagismo, A é a prevalência de consumo de álcool e (1-E) é o inverso da prevalência de excesso de peso, pois o último fator de risco é ausente nesse cluster. Foram entendidos como clusters todos os agrupamentos cuja razão O/E foi maior que 1 e cujo intervalo de 95\% de confiança não englobou a unidade 19,20,24. Assumiu-se que os fatores de risco ocorreram de maneira independente.

Análises descritivas foram realizadas para a caracterização da amostra por meio de frequências absolutas e relativas das variáveis com estratificação para sexo. Para as análises descritivas, foi utilizado o teste $\chi^{2}$ para testar as diferenças entre os sexos.

Para estimativa da probabilidade de se acumular um ou mais, dois ou mais, e três ou mais fatores de risco, foi utilizado o método de regressão ordinal apresentando a análise bruta e ajustada (o ajuste foi feito tendo em conta todas as variáveis de exposição) as respectivas odds ratio (OR) e seus IC95\%, utilizando como variáveis de exposição: sexo (masculino, feminino), idade (60-64, 65-69, 70-74, 75-79, $\geq 80$ ), cor de pele (branco, não branco), situação conjugal (com companheiro, sem com- 
panheiro) escolaridade (nenhuma, $\leq$ 4a série 1o grau, 1o grau completo, 2o grau completo ou mais), ocupação (ativo, não ativo) e nível econômico por um índice de bens (indicador econômico nacional IEN quintis) 34 .

Adicionalmente, foram realizadas analises de probabilidades condicionais, objetivando calcular a probabilidade de ocorrência de um fator de risco, na presença de outro. Sendo a OR representante da estimativa adicional que um comportamento pode ter em relação ao outro. Essa analise se deu mediante modelos de regressão logística com ajuste para as demais variáveis - sociodemográficas 14,19. Também foram apresentadas a fração etiológica $\left[\mathrm{FE}=(\% \text { exposição })^{*}(\mathrm{OR}-1) / 1+(\%\right.$ exposição $*(\mathrm{OR}-1)]$ e a fração prevenível $\left[\mathrm{FP}=(\% \text { exposição })^{*}(1-\mathrm{OR}) / \mathrm{OR}+(\% \text { exposição })^{*}(1-\mathrm{OR})\right]$ para essas análises condicionais.

As análises de prevalência observada e prevalência esperada, bem como a razão entre ambas e seus IC95\% foram realizadas com a utilização do software Microsoft Excel 2010 (Microsoft Corp., Estados Unidos). As demais análises foram realizadas no pacote estatístico Stata versão 12.1 (StataCorp LP, College Station, Estados Unidos). O efeito de delineamento amostral foi levado em consideração em todas as análises utilizando o comando survey (svy). Foi considerado um valor de $\mathrm{p}<0,05$ para significância estatística.

O estudo foi aprovado pelo Comitê de Ética em Pesquisa da Faculdade de Medicina da UFPel, em conjunto com os demais projetos pertencentes ao Consórcio de Pesquisa do Programa de Pós-graduação em Epidemiologia. A coleta de dados foi realizada perante esclarecimentos dos objetivos da pesquisa e possíveis riscos para os indivíduos, além da assinatura do Termo de Consentimento Livre e Esclarecido.

\section{Resultados}

Foram elegíveis para o estudo 1.844 idosos e, dentre eles, 1.451 foram entrevistados. O percentual de perdas e recusas foi $21,3 \%(n=393)$. As perdas e recusas não foram diferentes da amostra quanto a sexo, porém, quanto à idade, a amostra foi em média 1,2 anos mais velha $(\mathrm{p}=0,01)$.

A Tabela 1 descreve as características sociodemográficas, comportamentais e clínicas da amostra. Entre a população estudada, a proporção de um, dois e três ou mais fatores de risco foi de 37,2\%, 40,3\% e 10,6\%, respectivamente. Homens apresentaram maior prevalência de consumo de álcool (30,1\%; p < $0,001)$ e menor prevalência de inatividade física $(51 \%$; $<$ 0,001) em comparação a mulheres.

A Tabela 2 apresenta as prevalências observadas, esperadas e a razão O/E para as 16 combinações possíveis de fatores de risco. $\mathrm{O}$ agrupamento mais frequente foi inatividade física + excesso de peso (18,1\% nos homens e 30,7\% nas mulheres). Na análise de clusters, entre os homens, as combinações que apresentaram prevalência observada maior que a esperada foram: inatividade física + tabagismo $(\mathrm{O} / \mathrm{E}=1,51$; IC95\%: 1,06; 2,16) e consumo de álcool + excesso de peso (O/E = 1,27; IC95\%: 1,01; 1,59). Entre as mulheres, foram considerados clusters, inatividade física + tabagismo + consumo de álcool $(\mathrm{O} / \mathrm{E}=2,13$; IC95\%: 1,21; 3,76) e consumo de álcool + excesso de peso (O/E = 1,72; IC95\%: 1,35; 2,20) (Tabela 2).

As associações entre a simultaneidade de fatores de risco de acordo com as características da população são apresentadas na Tabela 3. Após os ajustes, apenas a idade foi associada inversamente à presença de mais fatores de risco. Idosos com 80 anos ou mais apresentaram, em média, 45\% menor chance de desenvolver mais um fator de risco (OR = 0,55; IC95\%: 0,39; 0,78).

A Tabela 4 apresenta a chance de um fator de risco estar associado a outro, dada a presença do primeiro. Indivíduos inativos apresentaram maior chance de ter excesso de peso $(\mathrm{OR}=1,52)$ e menor chance de ingerir álcool $(\mathrm{OR}=0,74)$, mesmo após ajustes para variáveis sociodemográficas. Além disso, indivíduos tabagistas apresentaram menor chance de apresentar excesso de peso $(\mathrm{OR}=0,61)$. As frações atribuíveis dessas associações também são apresentadas na Tabela 4. Verifica-se que ao eliminar a inatividade física da população, a prevalência de excesso de peso reduziria em $23,8 \%$. 


\section{Tabela 1}

Características da amostra de acordo com variáveis sociodemográficas, comportamentais e clínicas, 2014 ( $n=1.451$ ).

\begin{tabular}{|c|c|c|c|c|}
\hline Características & $\begin{array}{l}\text { Total } \\
\text { n (\%) }\end{array}$ & $\begin{array}{c}\text { Masculino } \\
\text { n (\%) }\end{array}$ & $\begin{array}{c}\text { Feminino } \\
\text { n (\%) }\end{array}$ & Valor de $p$ * \\
\hline Idade (anos) & & & & 0,412 \\
\hline $60-64$ & $394(27,2)$ & $158(29,5)$ & $236(25,9)$ & \\
\hline $65-69$ & $362(25,1)$ & $131(24,4)$ & $231(25,4)$ & \\
\hline $70-74$ & $270(18,7)$ & $103(19,2)$ & $167(18,3)$ & \\
\hline $75-79$ & $190(13,1)$ & $69(12,9)$ & $121(13,3)$ & \\
\hline$\geq 80$ & $230(15,9)$ & $75(14,0)$ & $155(17,0)$ & \\
\hline Cor da pele & & & & 0,331 \\
\hline Branco & $1.211(83,7)$ & $454(84,7)$ & $757(83,1)$ & \\
\hline Não branco & $236(16,3)$ & $82(15,3)$ & $154(16,9)$ & \\
\hline Situação conjugal & & & & $<0,001$ \\
\hline Com companheiro & $763(52,7)$ & $408(76,1)$ & $355(39,0)$ & \\
\hline Sem companheiro & $684(47,3)$ & $128(23,9)$ & $556(61,0)$ & \\
\hline Escolaridade & & & & 0,016 \\
\hline Nenhuma & $196(13,6)$ & $58(10,9)$ & $138(15,3)$ & \\
\hline$\leq 4 a$ a série do 1 o grau & $782(54,4)$ & $316(59,3)$ & $466(51,5)$ & \\
\hline 1 grau completo & $143(9,9)$ & $55(10,3)$ & $88(9,7)$ & \\
\hline 2o grau completo ou mais & $316(22,1)$ & $104(19,5)$ & $212(23,4)$ & \\
\hline Ocupação & & & & $<0,001$ \\
\hline Ativo & $264(19,6)$ & $144(27,1)$ & $120(14,7)$ & \\
\hline Não ativo & $1.084(80,4)$ & $387(72,9)$ & $697(85,3)$ & \\
\hline Indicar econômico nacional (quintis) & & & & 0,165 \\
\hline Q1 (mais pobre) & $282(20,5)$ & $94(18,5)$ & $188(21,7)$ & \\
\hline Q2 & $269(19,6)$ & $97(19,1)$ & $172(19,9)$ & \\
\hline Q3 & $275(20,0)$ & $98(19,3)$ & $177(20,5)$ & \\
\hline Q4 & $274(20,0)$ & $110(21,7)$ & $164(19,0)$ & \\
\hline Q5 (mais rico) & $273(19,9)$ & $109(21,5)$ & $164(19,0)$ & \\
\hline Fumo & & & & 0,108 \\
\hline Fumante & $182(12,6)$ & $76(14,2)$ & $106(11,6)$ & \\
\hline Não fumante & $1.264(87,4)$ & $460(85,8)$ & $804(88,4)$ & \\
\hline Consumo de álcool & & & & $<0,001$ \\
\hline Sim & $307(21,3)$ & $161(30,1)$ & $146(16,0)$ & \\
\hline Não & $1.138(78,7)$ & $374(69,9)$ & $764(84,0)$ & \\
\hline Índice de massa corporal & & & & 0,005 \\
\hline Eutrófico & $597(43,8)$ & $243(48,5)$ & $354(41,0)$ & \\
\hline Excesso de peso & $767(56,2)$ & $258(51,5)$ & $509(59,0)$ & \\
\hline Atividade física & & & & $<0,001$ \\
\hline Inativo & $824(60,1)$ & $257(51,0)$ & $567(65,3)$ & \\
\hline Ativo & $548(39,9)$ & $247(49,0)$ & $301(34,7)$ & \\
\hline Fatores simultâneos & & & & 0,133 \\
\hline 0 & $159(11,9)$ & $69(14,2)$ & $90(10,7)$ & \\
\hline 1 & $494(37,2)$ & $180(37,1)$ & $314(37,2)$ & \\
\hline 2 & $535(40,3)$ & $180(37,1)$ & $355(42,1)$ & \\
\hline 3 & $141(10,6)$ & $56(11,5)$ & $85(10,0)$ & \\
\hline
\end{tabular}

* Valor de p para heterogeneidade por sexo. 
Tabela 2

Prevalência e associação dos quatro fatores de risco estratificados por sexo, 2014 ( $n=1.329)$.

\begin{tabular}{|c|c|c|c|c|c|c|c|c|c|c|}
\hline \multirow{2}{*}{$\begin{array}{l}\text { Número de } \\
\text { fatores }\end{array}$} & \multirow[t]{2}{*}{ I } & \multirow[t]{2}{*}{ EP } & \multirow[t]{2}{*}{$\mathbf{F}$} & \multirow[t]{2}{*}{ A } & \multicolumn{3}{|c|}{ Masculino } & \multicolumn{3}{|c|}{ Feminino } \\
\hline & & & & & $\mathrm{O}(\%)$ & E (\%) & O/E (IC95\%) & $\mathrm{O}(\%)$ & $E(\%)$ & O/E (IC95\%) \\
\hline 4 & + & + & + & + & 1,7 & 1,1 & $1,47(0,87 ; 2,49)$ & 0,8 & 0,7 & $1,15(0,68 ; 1,96)$ \\
\hline 3 & + & + & + & - & 1,4 & 2,6 & $0,55(0,35 ; 0,86)$ & 3,2 & 3,8 & $0,85(0,66 ; 1,09)$ \\
\hline 3 & + & + & - & + & 6,2 & 6,8 & $0,91(0,72 ; 1,16)$ & 4,5 & 5,4 & $0,83(0,67 ; 1,02)$ \\
\hline 3 & + & - & + & + & 0,6 & 1,1 & $0,59(0,29 ; 1,17)$ & 1,1 & 0,5 & $2,13(1,21 ; 3,76)$ \\
\hline 3 & - & + & + & + & 1,7 & 1,1 & $1,53(0,90 ; 2,61)$ & 0,5 & 0,4 & $1,23(0,60 ; 2,52)$ \\
\hline 2 & + & + & - & - & 18,1 & 15,8 & $1,15(0,99 ; 1,34)$ & 30,7 & 28,6 & $1,07(0,99 ; 1,17)$ \\
\hline 2 & + & - & - & + & 4,3 & 6,4 & $0,68(0,52 ; 0,89)$ & 2,1 & 3,8 & $0,56(0,42 ; 0,75)$ \\
\hline 2 & + & - & + & - & 3,7 & 2,5 & $1,51(1,06 ; 2,16)$ & 2,7 & 2,6 & $1,04(0,78 ; 1,38)$ \\
\hline 2 & - & - & + & + & 1,0 & 1,0 & $1,01(0,55 ; 1,85)$ & 0,4 & 0,3 & $1,35(0,58 ; 3,15)$ \\
\hline 2 & - & + & + & - & 1,7 & 2,5 & $0,66(0,43 ; 1,02)$ & 1,2 & 2,0 & $0,59(0,40 ; 0,86)$ \\
\hline 2 & - & + & - & + & 8,3 & 6,5 & $1,27(1,01 ; 1,59)$ & 5,0 & 2,9 & $1,72(1,35 ; 2,20)$ \\
\hline 1 & + & - & - & - & 14,2 & 14,8 & $0,96(0,82 ; 1,13)$ & 19,9 & 19,9 & $1,00(0,90 ; 1,11)$ \\
\hline 1 & - & + & - & - & 13,0 & 15,1 & $0,86(0,73 ; 1,01)$ & 13,3 & 15,2 & $0,87(0,77 ; 0,99)$ \\
\hline 1 & - & - & + & - & 2,3 & 2,4 & $0,96(0,64 ; 1,44)$ & 1,9 & 1,4 & $1,36(0,94 ; 1,96)$ \\
\hline 1 & - & - & - & + & 7,6 & 6,1 & $1,24(0,98 ; 1,57)$ & 2,1 & 2,0 & $1,06(0,77 ; 1,47)$ \\
\hline 0 & - & - & - & - & 14,2 & 14,3 & $1,00(0,85 ; 1,17)$ & 10,7 & 10,6 & $1,01(0,88 ; 1,17)$ \\
\hline
\end{tabular}

A: consumo abusivo de álcool; E: valor esperado; EP: excesso de peso; F: dependência ao tabaco; I: inativo fisicamente; O: valor observado; O/E: observado/esperado.

Nota: em negrito, aqueles que foram considerados clusters.

\section{Discussão}

Dentre os fatores de risco avaliados neste estudo, o mais prevalente foi a inatividade física (60,1\%), e o menos frequente, o tabagismo (12\%). Mais de 50\% dos idosos apresentaram ao menos dois fatores de risco concomitantes. As combinações inatividade física + tabagismo e consumo de álcool + excesso de peso ocorreram mais do que o esperado ao acaso entre homens. Nas mulheres, os clusters mais frequentes foram inatividade física + tabagismo + consumo de álcool e consumo de álcool + excesso de peso. As combinações que ocorreram com menor frequência que o esperado entre os homens foram inatividade física + excesso de peso + tabagismo e inatividade física e consumo de álcool. Entre as mulheres foram excesso de peso + tabagismo e apenas excesso de peso. Além disso, excesso de peso e inatividade física parecem influenciar um ao outro assim como excesso de peso e tabagismo.

Alguns estudos sobre fatores de risco para doenças crônicas estão publicados 19,20,21,23, porém um número reduzido avaliou apenas idosos. Dentre os que fizeram esse tipo de análise, é possível destacar estudos em países da Europa, em sua maioria 20,22,24, estudos com diferentes faixas etárias - particularmente adultos - e também, na maioria das vezes, estudos que avaliam a dieta inadequada 19,20,23,24. Sendo assim, a maior idade dos sujeitos avaliados e a utilização do excesso de peso ao invés de dieta inadequada são fatores que dificultam as comparações com demais estudos.

No presente estudo, mais da metade dos idosos apresentou dois ou mais fatores de risco. Embora a comparação com outros estudos seja difícil, pois cada trabalho utiliza diferentes fatores de risco para mensurar a simultaneidade, a maioria apontou que a presença de mais de um fator de risco é alta, entre $40 \%$ e $60 \%$ 35,36,37,38. Ferreira et al. 25 encontraram prevalência superior a $80 \%$, entretanto este estudo incluiu somente idosos usuários do SUS, o que poderia justificar a elevada prevalência.

Para confirmar os resultados encontrados, realizou-se análise de componentes principais (dados não apresentados) para investigar os agrupamentos de fatores de risco, e os achados foram muito semelhantes aos encontrados com a presente análise. Dos estudos que abordam o tema da simultaneidade, a maioria encontrou clusters de inatividade física + dieta inadequada como os principais fatores 


\section{Tabela 3}

Associação entre a simultaneidade (dois ou mais) de fatores de risco de acordo com características sociodemográficas, $2014(n=1.329)$.

\begin{tabular}{|c|c|c|c|c|c|}
\hline \multirow[t]{2}{*}{ Características } & \multirow[t]{2}{*}{$\mathbf{n}$} & \multicolumn{4}{|c|}{$\begin{array}{l}\text { Fatores simultâneos } \\
\text { OR (IC95\%) }\end{array}$} \\
\hline & & Bruto & Valor de $p$ * & Ajustado & Valor de p * \\
\hline Sexo & 1.329 & & 0,263 & & 0,113 \\
\hline Masculino & & 1,00 & & 1,00 & \\
\hline Feminino & & $1,13(0.91 ; 1,42)$ & & $1,22(0,95 ; 1,58)$ & \\
\hline Idade (anos) & 1.329 & & $<0,001$ & & 0,001 \\
\hline $60-64$ & & 1,00 & & 1,00 & \\
\hline $65-69$ & & $0,80(0,61 ; 1,04)$ & & $0,82(0,62 ; 1,08)$ & \\
\hline $70-74$ & & $0,74(0,56 ; 0,99)$ & & $0,72(0,52 ; 0,99)$ & \\
\hline $75-79$ & & $0,65(0,47 ; 0,90)$ & & $0,65(0,45 ; 0,95)$ & \\
\hline$\geq 80$ & & $0,58(0,45 ; 0,75)$ & & $0,55(0,39 ; 0,78)$ & \\
\hline Cor de pele & 1.329 & & 0,365 & & 0,304 \\
\hline Branco & & 1,00 & & 1,00 & \\
\hline Não Branco & & $1,16(0,83 ; 1,61)$ & & $1,20(0,85 ; 1,69)$ & \\
\hline Situação conjugal & 1.329 & & 0,667 & & 0,795 \\
\hline Com companheiro & & 1,00 & & 1,00 & \\
\hline Sem companheiro & & $1,05(0,84 ; 1,32)$ & & $1,04(0,78 ; 1,37)$ & \\
\hline Escolaridade & 1.319 & & 0,763 & & 0,358 \\
\hline Nenhuma & & 1,00 & & 1,00 & \\
\hline$\leq 4$ a série do1o grau & & $1,12(0,85 ; 1,46)$ & & $1,07(0,80 ; 1,41)$ & \\
\hline 1o grau completo & & $0,96(0,64 ; 1,44)$ & & $0,90(0,58 ; 1,39)$ & \\
\hline 2o grau completo & & $1,07(0,75 ; 1,51)$ & & $0,86(0,55 ; 1,33)$ & \\
\hline Ocupação & 1.245 & & 0,054 & & 0,285 \\
\hline Ativo & & 1,00 & & 1,00 & \\
\hline Não ativo & & $1,33(0,99 ; 1,77)$ & & $1,19(0,86 ; 1,65)$ & \\
\hline Renda (IEN) & 1.263 & & 0,613 & & 0,313 \\
\hline 1 & & 1,00 & & 1,00 & \\
\hline 2 & & $1,15(0,84 ; 1,56)$ & & $1,19(0,86 ; 1,64)$ & \\
\hline 3 & & $1,07(0,78 ; 1,46)$ & & $1,15(0,83 ; 1,61)$ & \\
\hline 4 & & $0,97(0,72 ; 1,31)$ & & $1,01(0,74 ; 1,38)$ & \\
\hline 5 & & $1,21(0,88 ; 1,67)$ & & $1,36(0,93 ; 1,98)$ & \\
\hline
\end{tabular}

IC95\%: intervalo de 95\% de confiança; IEN: índice econômico nacional; OR: odds ratio.

* Valor de p para tendência linear. Análise ajustada para cada um dos fatores da tabela.

de risco para doenças crônicas 20,22,24. Neste estudo, a dieta inadequada não foi avaliada e o principal cluster encontrado foi excesso de peso + consumo de álcool tanto em homens quanto em mulheres como em outros estudos na Região Sul do Brasil 19,23. Estudos brasileiros, com adultos 23 e com adolescentes ${ }^{19}$, revelam que algumas combinações de fatores de risco como as combinações inatividade física + dieta inadequada e dieta inadequada + consumo de álcool parecem não se alterar ao longo do tempo. Isso pode ser explicado por hábitos como atividade física e alimentação que são estabelecidos na infância e tendem a perpetuar durante a vida adulta 39,40,41,42. Evidente que alimentação inadequada não é excesso de peso per se, mas a relação entre ambos é bem estabelecida 42 . Já o consumo de álcool, por ser um hábito que tem por característica a iniciação precoce no Brasil 14, também pode vir a ser mantido ao longo da vida. O presente estudo evidencia a ocorrência de clusters desses fatores de risco em idosos, podendo ser reflexo de hábitos da adolescência e início da vida adulta.

A formação dos clusters de fatores de risco em diferentes populações ocorre provavelmente em função das mudanças nas condições de vida ao longo dos anos, o que dificulta a manutenção de 
Tabela 4

Associação entre os fatores de risco (probabilidade para um fator se agrupar com outro), $2014(\mathrm{n}=1.362)$.

\begin{tabular}{|c|c|c|c|c|c|c|}
\hline \multirow[t]{2}{*}{ Fatores de risco } & \multicolumn{3}{|c|}{ Inatividade física } & \multicolumn{3}{|c|}{ Excesso de peso } \\
\hline & OR & IC95\% & FE & OR & IC95\% & FE \\
\hline Inatividade física & - & & - & 1,52 & 1,$18 ; 1,95$ & 23,8 \\
\hline Excesso de peso & 1,51 & 1,$18 ; 1,95$ & 22,3 & - & & - \\
\hline Consumo de álcool & 0,75 & 0,$56 ; 1,01$ & 6,6 & 1,31 & 0,$98 ; 1,76$ & 6,2 \\
\hline \multirow[t]{3}{*}{ Tabagismo } & 1,16 & 0,$81 ; 1,66$ & 2,0 & 0,61 & 0,$43 ; 0,87$ & 7,5 \\
\hline & \multicolumn{3}{|c|}{ Consumo de álcool } & \multicolumn{3}{|c|}{ Tabagismo } \\
\hline & OR & IC95\% & FE & OR & IC95\% & FE \\
\hline Inatividade física & 0,74 & 0,$55 ; 0,99$ & 17,4 & 1,14 & 0,$80 ; 1,64$ & 7,8 \\
\hline Excesso de peso & 1,29 & 0,$96 ; 1,73$ & 14,0 & 0,60 & 0,$42 ; 0,85$ & 27,3 \\
\hline Consumo de álcool & - & & - & 1,44 & 0,$96 ; 2,16$ & 8,6 \\
\hline Tabagismo & 1,44 & 0,$96 ; 2,16$ & 5,3 & - & & - \\
\hline
\end{tabular}

FE: fração etiológica; IC95\%: intervalo de 95\% de confiança; OR: odds ratio.

Nota: ajustado para variáveis sociodemográficas (sexo, idade, cor de pele, situação conjugal atual, escolaridade, status ocupacional, renda).

comportamentos saudáveis ${ }^{43}$. Atualmente, existem diversos obstáculos à manutenção do estilo de vida saudável, entre eles, a falta de tempo para preparo/alto custo de refeições adequadas, altas cargas de trabalho, profissões extremamente sedentárias, com nível elevado de estresse e uso exagerado de tecnologia além de outros fatores inerentes à evolução humana 44. Portanto, intervenções ou medidas preventivas focadas em mais de um fator de risco poderiam ser mais eficientes que aquelas focadas em apenas um.

A única variável associada à simultaneidade de fatores de risco no presente estudo foi a idade. Indivíduos mais velhos tiveram menor chance de apresentar mais de um fator de risco. Isso sugere a presença do viés de sobrevivência. Idosos acometidos por mais problemas de saúde, Talvez não estejam vivos, fato que acaba enviesando os resultados quanto à idade. Outra possibilidade relativa ao viés de sobrevivência é que esses idosos podem ter parado com alguns hábitos como fumo e álcool ou se tornaram inativos à medida que foram desenvolvendo limitações por causa de doenças.

Os resultados das probabilidades condicionais mostraram que ser inativo aumenta a chance de apresentar excesso de peso e vice-versa. Mesmo com a possível causalidade reversa, problema intrínseco ao estudo transversal, é provável que o acúmulo de inatividade física leve ao baixo gasto energético e consequente excesso de peso. As associações sugerem que intervir em ambos os comportamentos pode ser positivo no combate à simultaneidade dos fatores de risco. Por outro lado, constatou-se que o tabagismo diminuiu a chance de excesso de peso. Tal achado possivelmente ocorreu em função dos efeitos da nicotina no gasto energético 45 e consequente proteção para sobrepeso e obesidade. Cabe ressaltar, todavia, que os diversos malefícios do cigarro à saúde não se equiparam à redução do peso 46 .

Como pontos fortes do trabalho, ressalta-se que, por se tratar de um estudo de base populacional com amostragem probabilística e rigor metodológico, torna os resultados extrapoláveis para a população alvo (idosos de Pelotas). Além disso, a cidade tem aspecto socioeconômico que se assemelha a várias cidades de médio porte no Brasil e poucos estudos avaliaram este desfecho na faixa etária de idosos, população altamente suscetível ao acúmulo de fatores de risco.

Contudo, este estudo não é isento de limitações; entre elas, encontra-se a menor idade entre as perdas e recusas quando comparadas à amostra efetiva. Esse fato pode levar a uma subestimação das prevalências isoladas dos fatores de risco estudados e também para as análises de cluster porque as maiores frequências de simultaneidade encontradas estão entre os idosos do grupo de menor idade. 
Por se tratar de um estudo transversal, há a possibilidade de viés de sobrevivência, como comentado anteriormente, que pode ter subestimado as prevalências de tabagismo, consumo de álcool, inatividade física e excesso de peso. Ademais, as associações em relação ao consumo de álcool devem ser interpretadas com cautela. No presente estudo, não foi avaliada a dosagem de álcool ingerida ou dependência, mas somente o consumo nos últimos 30 dias. Isso acaba agrupando em uma mesma categoria tanto idosos que consomem altas doses quanto aqueles que consumiram pelo menos uma dose no último mês, superestimando essa prevalência. Por fim, medidas autorreferidas são sujeitas a viés de recordatório sobretudo em idades mais avançadas.

A simultaneidade de fatores de risco é um tema de grande importância, pois aqueles indivíduos que apresentam mais de um fator de risco estão em pior situação de saúde do que aqueles apresentam apenas um ou nenhum. A presença de dois ou mais fatores de risco em mais da metade da população idosa indica a necessidade de políticas públicas voltadas à promoção de um estilo de vida saudável, com a consequente redução esperada dos gastos desnecessários ao sistema de saúde. Estudos que avaliem o impacto da ocorrência simultânea de fatores de risco sobre doenças crônicas devem ser estimulados, focando a melhor compreensão desse fenômeno. As frações atribuíveis reforçam o impacto positivo que políticas e intervenções centradas em fatores de risco poderiam ter na população idosa.

Este estudo mostra que os idosos de Pelotas apresentam fatores de risco simultâneos. Repensar intervenções e políticas de combate a comportamentos prejudiciais à saúde poderia ter maior impacto na saúde da população de 60 anos ou mais.

\section{Colaboradores}

M. F. Cruz participou da concepção do projeto, interpretação dos dados, redação do artigo. V. V. Ramires, G. I. Mielke, J. Martinez-Mesa, F. C. Wehrmeister participaram da concepção do projeto, interpretação dos dados e revisão crítica do conteúdo. A. W. Böhm participou da interpretação dos dados e redação do artigo. Todos os autores aprovaram a versão final a ser publicada e são responsáveis por todos os aspectos do trabalho.

\section{Agradecimentos}

À PROEX e à Coordenação de Aperfeiçoamento Pessoal de Nível Superior (Capes) pelo suporte financeiro ao consórcio do mestrado da Universidade Federal de Pelotas. E também à Fundação de Amparo à Pesquisa do Estado do Rio Grande do Sul (FAPERGS) e à Capes pela concessão de bolsas durante o curso de mestrado.

\section{Referências}

1. Wong LLR, Carvalho JA. O rápido processo de envelhecimento populacional do Brasil: sérios desafios para as políticas públicas. Rev Bras Estud Popul 2006; 23:5-26.

2. Duarte EC, Barreto SM. Transição demográfica e epidemiológica: a Epidemiologia e Serviços de Saúde revisita e atualiza o tema. Epidemiol Serv Saúde 2012; 21:529-32.

3. Vasconcelos A, Gomes M. Transição demográfica: a experiência brasileira. Epidemiol Serv Saúde 2012; 21:539-48.

4. Instituto Brasileiro de Geografia e Estatística. Projeção da população do Brasil e das Unidades da Federação 2016. http://www.ibge.gov.br/ apps/populacao/projecao/ (acessado em Fev/ 2016).

5. Instituto Brasileiro de Geografia e Estatística. Perfil dos idosos responsáveis por domicílios no Brasil/2000. Rio de Janeiro: Instituto Brasileiro de Geografia e Estatística; 2002.

6. Ministério da Saúde. Atenção à saúde da pessoa idosa e envelhecimento. Brasília: Ministério da Saúde; 2006. (Série Pactos pela Saúde). (Série B, Textos Básicos de Saúde).

7. Batista Filho M, Rissin A. A transição nutricional no Brasil: tendências regionais e temporais. Cad Saúde Pública 2003; 19 Suppl 1:S181-91.

8. Reichenheim ME, Souza ER, Moraes CL, de Mello Jorge MH, da Silva CM, de Souza Minayo MC. Violence and injuries in Brazil: the effect, progress made, and challenges ahead. Lancet 2011; 377:1962-75.

9. World Health Organization. Global strategy to reduce the harmful use of alcohol. Geneva: World Health Organization; 2010.

10. World Health Organization. World health statistics 2013. Geneva: WHO Press; 2013. 
11. Wood DM, Mould MG, Ong SB, Baker EH. "Pack year" smoking histories: what about patients who use loose tobacco? Tob Control 2005; 14:141-2.

12. Lee IM, Shiroma EJ, Lobelo F, Puska P, Blair SN, Katzmarzyk PT. Effect of physical inactivity on major non-communicable diseases worldwide: an analysis of burden of disease and life expectancy. Lancet 2012; 380:219-29.

13. World Health Organization. Global status report on noncommunicable diseases 2010. Geneva: World Health Organization; 2011.

14. Schmidt MI, Duncan BB, Azevedo e Silva G, Menezes AM, Monteiro CA, Barreto SM, et al. Chronic non-communicable diseases in Brazil: burden and current challenges. Lancet 2011; 377:1949-61.

15. Malta DC, Andrade SSCA, Stopa SR, Pereira CA, Szwarcwald CL, Silva Júnior JB, et al. Estilos de vida da população brasileira: resultados da Pesquisa Nacional de Saúde, 2013. Epidemiol Serv Saúde 2015; 24:217-26.

16. Departamento de Vigilância de Doenças e Agravos Não Transmissíveis e Promoção da Saúde, Secretaria de Vigilância em Saúde, Ministério da Saúde. Vigitel Brasil 2013: Vigilância de Fatores de Risco e Proteção para Doenças Crônicas por Inquérito Telefônico. Brasília: Ministério da Saúde; 2014. (Série G. Estatística e Informação em Saúde).

17. Nigg CR, Allegrante JP, Ory M. Theory-comparison and multiple-behavior research: common themes advancing health behavior research. Health Educ Res 2002; 17:670-9.

18. Goldstein MG, Whitlock EP, DePue J. Multiple behavioral risk factor interventions in primary care. Summary of research evidence. Am J Prev Med 2004; 27(2 Suppl):61-79.

19. Dumith SC, Muniz LC, Tassitano RM, Hallal PC, Menezes AM. Clustering of risk factors for chronic diseases among adolescents from Southern Brazil. Prev Med 2012; 54:393-6.

20. Galan I, Rodriguez-Artalejo F, Tobias A, DiezGanan L, Gandarillas A, Zorrilla B. Clustering of behavior-related risk factors and its association with subjective health. Gac Sanit 2005; 19:370-8.

21. Muniz LC, Schneider BC, Silva IC, Matijasevich A, Santos IS. Accumulated behavioral risk factors for cardiovascular diseases in Southern Brazil. Rev Saúde Pública 2012; 46:534-42.

22. Poortinga W. The prevalence and clustering of four major lifestyle risk factors in an English adult population. Prev Med 2007; 44:124-8.

23. Silva DA, Peres KG, Boing AF, Gonzalez-Chica DA, Peres MA. Clustering of risk behaviors for chronic noncommunicable diseases: a population-based study in southern Brazil. Prev Med 2013; 56:20-4.

24. Schuit AJ, van Loon AJM, Tijhuis M, Ocké MC. Clustering of lifestyle risk factors in a general adult population. Prev Med 2002; 35:219-24.

25. Ferreira CCC, Peixoto MRG, Barbosa MA, Silveira EA. Prevalência de fatores de risco cardiovascular em idosos usuários do Sistema Único de Saúde de Goiânia. Arq Bras Cardiol 2010; 95:621-8.
26. Barros AJD, Menezes AMB, Santos IS, Assunção MCF, Gigante D, Fassa AG, et al. O Mestrado do Programa de Pós-graduação em Epidemiologia da UFPel baseado em consórcio de pesquisa: uma experiência inovadora. Rev Bras Epidemiol 2008; 11:133-44.

27. Instituto Brasileiro de Geografia e Estatística. Censo demográfico 2010. Rio de Janeiro: Instituto Brasileiro de Geografia e Estatística; 2013.

28. Centers for Disease Control and Prevention. Adult tobacco use information. http://www. cdc.gov/nchs/nhis/tobacco/tobacco_glossary. htm (acessado em Mar/2016).

29. Lipschitz DA. Screening for nutritional status in the elderly. Prim Care 1994; 21:55-67.

30. Chumlea W, Guo S, Steinbaugh M. Prediction of stature from knee height for black and white adults and children with application to mobility-impaired or handicapped persons. J Am Diet Assoc 1994; 94:1385-8.

31. Ritz P. Validity of measuring knee-height as an estimate of height in diseased French elderly persons. J Nutr Health Aging 2004; 8:386-8.

32. Craig CL, Marshall AL, Sjostrom M, Bauman AE, Booth ML, Ainsworth BE, et al. International physical activity questionnaire: 12 -country reliability and validity. Med Sci Sports Exerc 2003; 35:1381-95.

33. World Health Organization. Global recommendations on physical activity for health. Geneva: World Health Organization; 2010.

34. Barros AJD, Victora CG. Indicador econômico para o Brasil baseado no censo demográfico de 2000. Rev Saúde Pública 2005; 39:523-9.

35. Pereira JC, Barreto SM, Passos VMA. The profile of cardiovascular health of elderly brazilian people needs to improve: a population-based study. Arq Bras Cardiol 2008; 91:1-10.

36. Lessa I, Araújo MJ, Magalhães L, Almeida Filho N, Aquino E, Costa MCR. Simultaneidade de fatores de risco cardiovascular modificáveis na população adulta de Salvador (BA), Brasil. Rev Panam Salud Pública 2004; 16:131-7.

37. Barreto SM, Passos VMA, Firmo JOA, Guerra HL, Vidigal PG, Lima-Costa MFF. Hypertension and clustering of cardiovascular risk factors in a community in Southeast Brazil: the Bambuí Health and Ageing Study. Arq Bras Cardiol 2001; 77:576-81.

38. Fuchs SC, Moreira LB, Camey SA, Moreira MB, Fuchs FD. Clustering of risk factors for cardiovascular disease among women in Southern Brazil: a population-based study. Cad Saúde Pública 2008; 24 Suppl 2:S285-93.

39. Azevedo Júnior MR, Araújo CLP, Pereira FM. Atividades físicas e esportivas na adolescência: mudanças de preferências ao longo das últimas décadas. Rev Bras Educ Fís Esp 2006; 20:51-8.

40. Kvaavik E, Tell GS, Klepp KI. Predictors and tracking of body mass index from adolescence into adulthood: follow-up of 18 to 20 years in the Oslo Youth Study. Arch Pediatr Adolesc Med 2003; 157:1212-8. 
41. Eissa MA, Dai S, Mihalopoulos NL, Day RS, Harrist RB, Labarthe DR. Trajectories of fat mass index, fat free-mass index, and waist circumference in children: Project HeartBeat! Am J Prev Med 2009; 37(1 Suppl):S34-9.

42. Viner RM, Cole TJ. Who changes body mass between adolescence and adulthood? Factors predicting change in BMI between 16 year and 30 years in the 1970 British Birth Cohort. Int J Obes (Lond) 2006; 30:1368-74.

43. Jamison DT, Breman JG, Measham AR, Alleyne G, Claeson M, Evans DB, et al. Noncommunicable disease and injury disease control priorities in developing countries. 2nd Ed. Washington DC: The International Bank for Reconstruction and Development; The World Bank/ New York: Oxford University Press; 2006.

\begin{abstract}
This study aimed to describe the simultaneity of risk factors for chronic non-communicable diseases among the elderly ( $\leq 60$ years) in a city in Southern Brazil. This was a cross-sectional, population-based study of 1,451 elderly in 2013. Cluster analysis was applied to selected risk factors (smoking, alcohol consumption, excess weight, and physical inactivity). Logistic regression was used to assess the association between simultaneity of risk factors and socio-demographic variables. The most frequent cluster in men (18.1\%) and women $(30.7 \%)$ was physical inactivity + excess weight. The cluster alcohol consumption + excess weight exceeded the expected level in men $(\mathrm{O} / \mathrm{E}=$ 1.27; 95\%CI: $1.01 ; 1.59)$ and women $(\mathrm{O} / \mathrm{E}=$ 1.72; 95\%CI: $1.35 ; 2.20)$. The presence of two or more risk factors in the elderly population (88.1\%) points to the need for specific interventions for this population to fight risk factors simultaneously rather than separately.
\end{abstract}

Chronic Disease; Risk Factors; Aged
44. Lim SS, Vos T, Flaxman AD, Danaei G, Shibuya $\mathrm{K}$, Adair-Rohani $\mathrm{H}$, et al. A comparative risk assessment of burden of disease and injury attributable to 67 risk factors and risk factor clusters in 21 regions, 1990-2010: a systematic analysis for the Global Burden of Disease Study 2010. Lancet 2012; 380:2224-60.

45. Blauw LL, Boon MR, Rosendaal FR, de Mutsert R, Gast KB, van Dijk KW, et al. Smoking is associated with increased resting energy expenditure in the general population: the NEO study. Metabolism 2015; 64:1548-55.

46. Aveyard P, Lycett D, Farley A. Managing smoking cessation-related weight gain. Pol Arch Med Wewn 2012; 122:494-8.

\section{Resumen}

El objetivo del estudio fue describir la simultaneidad de factores de riesgo para enfermedades crónicas no transmisibles en ancianos (60 años o más), residentes en una ciudad del Sur de Brasil. Se trata de un estudio transversal, de base poblacional, realizado en 1.451 ancianos en 2013. Con un enfoque de análisis de clusters, fue evaluado el agrupamiento entre los factores de riesgo en un estudio (tabaquismo, consumo de alcohol, exceso de peso e inactividad física). Para la evaluación de la asociación de la simultaneidad de los factores de riesgo con variables sociodemográficas, se utilizó la regresión logística. El agrupamiento más frecuente entre los hombres (18.1\%) y mujeres (30,7\%) fue inactividad física + exceso de peso. Las combinaciones consumo de alcohol + exceso de peso excedieron lo esperado entre los hombres $(O / E=1,27$; IC95\%: 1,01; 1,59) y mujeres $(\mathrm{O} / \mathrm{E}=1,72$; IC95\%: 1,35; 2,20). La presencia de dos o más factores de riesgo en la población anciana $(88,1 \%)$ apunta la necesidad de intervenciones específicas para esta población dirigidas al combate simultáneo de los factores de riesgo y no de forma aislada.

Enfermedad Crónica; Factores de Riesgo; Anciano
Recebido em 12/Fev/2016

Versão final reapresentado em 28/Mar/2016

Aprovado em 02/Mai/2016 